\title{
Experimental Tests of Neutron Shielding for the ATLAS Forward Region
}

S. Pospíšil, I. Štekl, T. Čechák, P. Čermák, J. Jakůbek, J. Klusoň,

J. Koníček, J. Kubašta, V. Linhart, M. Šiňor

Faculty of Nuclear Sciences and Physical Engineering

Czech Technical University, Prague

C. Leroy

Physics Department, University of Montreal, Montreal

Z. Doležal, R. Leitner, G. Lukhanin, K. Soustružník

Nuclear Center, Faculty of Mathematics and Physics

Charles University, Prague

M. Lokajíček, S. Němeček

Institute of Physics

Academy of Sciences of the Czech Republic, Prague

J. Palla, J. Sodomka

Faculty of Transportation Sciences

Czech Technical University, Prague

August 13, 1998

\begin{abstract}
Experimental tests devoted to the optimization of the neutron shielding for the ATLAS forward region were performed at the CERN-PS with a $4 \mathrm{GeV} / \mathrm{c}$ proton beam. Spectra of fast neutrons, slow neutrons and gamma rays escaping a block of iron $(40 \times 40 \times 80$ $\mathrm{cm}^{3}$ ) shielded with different types of neutron and gamma shields (pure polyethylene - PE, borated polyethylene - BPE, lithium filled polyethylene - LiPE, lead, iron) were measured by means of plastic scintillators, a Bonner spectrometer, a HPGe detector and a slow neutron detector. Effectiveness of different types of shielding against neutrons and $\gamma$-rays were compared. The idea of a segmented outer layer shielding (iron, BPE, iron, LiPE) for the ATLAS Forward Region was also tested.
\end{abstract}

\section{Introduction}

The head-on collisions of $7 \mathrm{TeV}$ protons at the Large Hadron Collider ( $\mathrm{LHC}$ ) [1] at the expected peak luminosity of $1.0 \times 10^{34} \mathrm{~cm}^{-2} \mathrm{sec}^{-1}$ will produce high fluences of particles at the interaction point. The dominant source of radiation will be from neutrons and photons copiously produced in the high rate proton-proton collisions at large impact parameter

${ }^{*}$ Corresponding author. E-mail: stekl@br.fjfi.cvut.cz. 
and subsequent hadron interactions in the surrounding material. High-energy particles from the interaction point begin to cascade when entering material. If the material is thick, the cascade development will continue until most of the charged particles have been absorbed. For such a purpose, this material has to be as dense as possible. The remnants are mostly neutrons and associated gamma. While electromagnetic cascade are rapidly absorbed, neutrons will travel long distance losing their energy gradually. Nuclear capture of thermal neutrons frequently results into production of gamma. Gamma also result from excited-state decay of spallation products and from fast neutron interactions with atomic nuclei. Most of them, neutrons and gamma, should be confined in the shielding volume. The ATLAS forward region has to be surrounded by a structure made of neutron and gamma absorbing materials selected and combined towards optimal moderation of unconfined fast neutrons and reduction of gamma radiation produced by neutrons during the slowing down process in the shielding materials themselves. The building of a shield against fast neutrons proceeds according to a two-step golden rule: i) first moderate, ii) then capture. This rule has been kept as a guidance in the present studies.

The innermost layer of the shielding in the ATLAS forward region [2] naturally acts as an hadron shield. It should have a short hadronic interaction length but should not produce a copious number of secondary neutrons. Therefore, iron is used as the main hadron shielding material. The hadron shielding cone is surrounded with a neutron shield made of polyethylene (PE) [2] in which fast secondary neutrons are effectively moderated and partially captured through elastic scattering and radiative capture by hydrogen. In the same reference, a proposal was also made to surround a polyethylene layer with an additional layer of lead $5 \mathrm{~cm}$ thick to shield against photons from neutron capture.

Another proposal to shield against fast neutrons inside the ATLAS apparatus was based on the use of borated polyethylene (BPE) [3]. Hydrogen contained in PE is responsible for neutron slowing down while boron is added to capture the slowed neutrons. This solution was affording the possibility to prevent transport of neutrons and production of highly energetic gamma-rays from neutron radiative capture in surrounding materials (e.g. $7631 \mathrm{keV}, 7645 \mathrm{keV}$ in Fe; $7914 \mathrm{keV}, 7637 \mathrm{keV}$ in $\mathrm{Cu} ; 4934 \mathrm{keV}, 3935 \mathrm{keV}$ in Si) or in $\mathrm{PE}$ itself mainly due to the ${ }^{1} \mathrm{H}(\mathrm{n}, \gamma)^{2} \mathrm{H}$ reaction with a gamma energy $E_{\gamma}=2223$ $\mathrm{keV}$, thus suppressing the ATLAS detector background signal, significantly. However, the proposal of a boron based shield suffers from a major drawback which is the gamma-ray production from the reaction ${ }^{10} \mathrm{~B}(\mathrm{n}, \alpha)^{7} \mathrm{Li}^{*} \rightarrow{ }^{7} \mathrm{Li}+\gamma, E_{\gamma}=478 \mathrm{keV}$, the dominant channel after neutron absorption.

To avoid the disadvantage inherent to boron based shielding, it was suggested [4] to use as a neutron shield lithium doped polyethylene (LiPE) where the reaction responsible for neutron capture is ${ }^{6} \mathrm{Li}(\mathrm{n}, \alpha)^{3} \mathrm{H}$ with no gamma-ray production.

The goal of the present work was to put to the test in a beam environment of the CERN proton-synchrotron (CERN-PS) facility this idea of lithium based shielding and to extent comparison with boron-based shielding. It was also motivated by the idea to avoid extensive use of lead in the outer layer of the forward region shielding. It would make the shielding cheaper and possibly simplify the problem of construction.

The conditions expected on the surface of the ATLAS forward hadron shielding [2] were simulated experimentally with the help of an iron block exposed to a proton beam at the CERN-PS. The measurements lead to the proposal of a segmented outer layer shielding (iron, BPE, iron, LiPE). The possibility to extend this solution to other situations such as the application of combined neutron and gamma shielding to underground experiments (e.g. [5]) is mentioned. 


\section{Experimental procedure}

The measurements were performed at the secondary beam t10 of the CERN-PS facility. The experimental set-up is schematically shown in Fig. 1 and 2. The environment of neutrons and gamma against which various shieldings are tested is produced with an iron block of dimensions of $40 \mathrm{~cm} \times 40 \mathrm{~cm} \times 80 \mathrm{~cm}$, struck by a $4 \mathrm{GeV} / \mathrm{c}$ collimated proton beam. The block was shielded on one side by different types of neutron and/or gamma shields. The distance between the beam and the shielded surface of the iron block was selected as $25 \mathrm{~cm}$. The beam cycle was approximately $14 \mathrm{~s}$ with one or two burst each of about $400 \mathrm{~ms}$ of duration. The data acquisition system was gated in coincidence with the proton burst (time window $\sim 700 \mathrm{~ms}$ ) to decrease the counting of unwanted background radiation and muons.

The number of protons was monitored by means of a standard PS beam wire chamber and by two scintillator detectors (S6, S7) with an overlapping surface of $10 \times 20 \mathrm{~cm}^{2}$ standing in the beam in front of the iron block ( Fig. 2 ). Independent integral monitoring of the number of hadronic cascades produced in the iron block was done by means of a BGO spectroscopic scintillator positioned on the top of the iron block (Fig. 1) sensitive to the electromagnetic component of the cascade.

Precise gamma-ray spectroscopy performed with a HPGe detector gave exhaustive information about the origin of the detected gamma-rays. The HPGe detector was covered by a $\mathrm{Cd}$ foil $0.5 \mathrm{~mm}$ thick. The following gamma-rays were monitored:

$478 \mathrm{keV}$ from the ${ }^{10} \mathrm{~B}(\mathrm{n}, \alpha)$ reaction,

$558 \mathrm{keV}$ from the ${ }^{113} \mathrm{Cd}(\mathrm{n}, \gamma)$ reaction as a measure of the thermal neutron flux,

$847 \mathrm{keV}$ from the ${ }^{56} \mathrm{Fe}\left(\mathrm{n}, \mathrm{n}^{\prime} \gamma\right)$ reaction as a measure of the number of inelastic scattering of the fast neutrons in the iron block itself,

$2223 \mathrm{keV}$ from the ${ }^{1} \mathrm{H}(\mathrm{n}, \gamma)$ reaction as a measure of the number of thermal neutrons captured by hydrogen in any type of polyethylene,

$7631+7645 \mathrm{keV}$ doublet from the ${ }^{56} \mathrm{Fe}(\mathrm{n}, \gamma)$ reaction as a measure of the number of thermal neutrons captured in the iron block. Thermal neutrons were produced via slowing down of neutrons in the block itself and in the surrounding materials (polythylene neutron shield and concrete present in the experimental zone).

Fast neutron spectra were measured with a Bonner spectrometer [6]. It consists of a thermal neutron detector, ${ }^{6} \mathrm{LiI}(\mathrm{Eu})$ crystal, centered in polyethylene moderating spheres having diameters from 5 up to $25 \mathrm{~cm}$. The neutron flux energy distributions were obtained using methods described in refs. [7] and [8]. The fluence responses of the spheres were based on experimental calibrations performed with a ${ }^{252} \mathrm{Cf}$ neutron source. The contribution of the $\mu$ component was measured during beam-off periods and substracted from the fluence responses. Thermal neutron flux from shielding was monitored by means of a Si detector in connection with a ${ }^{6} \mathrm{Li}$ converter [9].

The charged particles escaping the iron block were registered by means of a wall of four large organic scintillator detectors, each made of a $2 \mathrm{~cm}$ thick polystyrene plate [10] that covered one side-wall of the target iron block with an active area of $40 \times 80 \mathrm{~cm}^{2}$ (Fig. 2 ). The detectors have been numbered as S1 (the most upstream detector) to $\mathrm{S} 4$. One scintillator detector (S5) was placed behind the iron block to monitor muon contamination of the beam $(\sim 15 \%)$. Signals from detectors S5, S6 and S7 were led to a coincidence module where the gate signal was generated according to a $\overline{S 5} \times \mathrm{S} 6 \times \mathrm{S} 7$ criterion. The signals from the scintillator detectors S1-S4 were led to charge integrating analog-to-digital converters ( ADC, LeCroy 2249A).

Neutrons and $\gamma$-rays also produce signals in the scintillating detectors. In order to minimize pile-up effect, the signal from scintillating detectors has been integrated during $50 \mathrm{~ns}$ long gate by charge ADC. Such a short gate however disabled the detection of neutrons with kinetic energies below about $0.3 \mathrm{MeV}$ and of $\gamma$-rays produced by the neutron 
capture in shielding material. The event by event charge response was registered via an acquisition system based on a PC computer and CAMAC controller. The charged spectra were analysed off-line. The number of hits above threshold for each of the four detectors S1-S4 was extracted. For the analysis, the threshold has been set to 3 ADC channels above pedestal, i.e. about $4 \%$ of the minimum ionizing particle (mip) signal. The signal of mip corresponding to $4 \mathrm{MeV}$ of losses in the scintillators has been adjusted to 75 ADC channels. The responses of the detectors S1-S4 have been equalized within $\pm 10 \%$ using beam particles.

\section{Analysis of experimental data}

The first two runs were devoted to the estimate of the number of neutrons and $\gamma$-rays yielded by proton interactions in the iron block and to assess the basic qualities of all types of neutron shielding (PE, BPE, LiPE) from the point of view of the $\gamma$-ray production and the influence on the neutron flux.

First of all, tests were performed to determine the distance between the hitting point of the $4 \mathrm{GeV} / \mathrm{c}$ proton beam into the iron block and the edge of the iron block which has to be shielded against neutrons. The distance of $25 \mathrm{~cm}$ was selected after combining the informations from these test measurements (Table 1), standard calorimetry formulae about development of hadron cascades [11] and availability of space in the experimental set-up.

The bulk of measurements during the second run was performed with different types of polyethylene (PE, BPE, LiPE) and different thicknesses $(8 \mathrm{~cm}$ and $16 \mathrm{~cm}$ ) of neutron shielding. These neutron shieldings were produced in KOPOS Kolín (Czech Republic). The chemical composition of $\mathrm{PE}$ is $\mathrm{CH}_{2}$, $\mathrm{BPE}$ is $\mathrm{CH}_{2}+\mathrm{H}_{3} \mathrm{BO}_{3}$ (3 weight \% of $\mathrm{B}$ ) and LiPE is $\mathrm{CH}_{2}+\mathrm{Li}_{2} \mathrm{CO}_{3}$ (10 weight \% of Li) [4].

The results of gamma-ray measurements from the second run are summarized in Table 2. The results clearly display a significant suppression of neutron capture gamma rays (2223 keV, $7631 \mathrm{keV}, 7645 \mathrm{keV}$ ) by both types of doped PE (BPE, LiPE) compared with pure PE. The disadvantage of BPE compared to LiPE is illustrated by the huge amount of gamma-rays accompanying neutron absorption by boron (column 3 of the Table $2, \mathrm{E}_{\gamma}$ $=478 \mathrm{keV}$ ), which would also require additional gamma shield.

Measurement with the unshielded iron block was performed to estimate the yield of neutrons and gamma directly produced as a result of proton cascades in the iron block itself and to estimate the level of the background radiation. This background radiation was resulting from the interaction of surrounding materials (concrete, magnets, etc...) in the experimental hall with neutrons escaping the iron block and with the variety of particles from the accelerator environment. Background radiation influenced all measurements. The $847 \mathrm{keV}$ spectral line from iron gives information about the presence of fast neutrons (Table 2) .

A third run of measurements was performed to compare $\mathrm{Fe}$ and $\mathrm{Pb}$ gamma-ray shielding capability and then to test the merits of the segmented shielding (BPE, Fe, LiPE). The comparison between $\mathrm{Fe}$ and $\mathrm{Pb}$ gamma-ray shielding was based on measurements of $\gamma$-ray yields from the whole set-up with $16 \mathrm{~cm}$ of BPE. The thickness of $\mathrm{Fe}$ and $\mathrm{Pb}$ shielding was $5 \mathrm{~cm}$. The results are presented in Table 3 . One can see from this table that low energy $\gamma$-ray production which takes place in BPE is significantly suppressed even by iron shielding. It indicates that iron can be an alternative to the originally proposed choice of lead [2].

As a next step, an idea of segmented shielding was tested. Firstly, measurements were performed with a configuration made of a BPE layer $(8 \mathrm{~cm}$ thick) and various thicknesses of iron (from 0 to $8 \mathrm{~cm}$ ). This set-up gives the information about the amount 
of $\gamma$-rays confined inside this configuration. The results are presented in Table 4. In this configuration still are outgoing fast neutrons, and slow neutrons remaining from the shielding itself, and incoming neutrons from the experimental zone environment. The latter contribution is expected to be especially important in the case of ATLAS since neutrons internally produced in the other parts of the detector can enter the shielding cone. Secondly, with the aim to remove this possibly large fraction of residual neutrons and to prevent their interaction with the iron shielding layer without $\gamma$-ray production, a layer of LiPE neutron shielding, $8 \mathrm{~cm}$ thick, was added to the configuration above. The results of neutron and $\gamma$-ray measurements for the combination of BPE, iron, LiPE shielding layers, hereafter called segmented shielding, are reported in Table 5. From these results, it can be observed that additional suppression of the detected radiation takes place.

The results obtained from Bonner spectrometer measurements are shown in Figs. 3 and 4. The neutron flux energy distribution for different types of neutron shielding materials ( PE, BPE, LiPE ) and for the non-shielded iron block are compared in Fig. 3. The thickness of the neutron shielding material was $16 \mathrm{~cm}$ in all cases. For all the shielding materials considered, a neutron flux suppression is significant compared with non-shielded iron. The comparison between the neutron fluxes of the originally proposed shielding [2] ( $\mathrm{PE}$ and $5 \mathrm{~cm}$ thick $\mathrm{Pb}$ layer ) and shielding consisting of $\mathrm{BPE}$ and iron is presented in Fig. 4. It is seen from that figure that the shielding proposed in the present work gives lower neutron flux compared with the shielding based on $\mathrm{PE}$ and $\mathrm{Pb}$. Such an effect could be explained by spallation neutrons from lead.

Some information about the lateral profile of cascades and associated radiation was obtained with the help of the scintillating wall. Tables 6 and 7 show the dependence of the scintillator wall signal (S1-S4) on various types of neutron shielding (PE, BPE, LiPE, each with a thickness of $8 \mathrm{~cm}$ ) placed between the scintillator wall and the iron block, as well as comparing with the results obtained from measurement without any neutron shielding. The results are given for two distances $(15 \mathrm{~cm}$ and $\sim 25 \mathrm{~cm}$ ) between the hitting point of the proton beam into the iron block and the edge of the iron block on the side of the scintillating wall. The values show the effectiveness of the neutron shielding. From the point of view of the signal obtained from the scintillator detectors S1-S4, both types of neutron shielding (BPE, LiPE) are comparable and are more effective than PE.

An attempt was done to reproduce these results by Monte Carlo simulations. The measurements for a $5 \mathrm{GeV} / \mathrm{c}$ proton beam and an iron block thickness of $20 \mathrm{~cm}$ have been compared with GEANT [12] simulations. The comparisons of spectra from the scintillator detectors S1-S4 are shown in Fig. 5 and 6 . The simulated and experimental spectra agree quite well. However, in the higher energy region the simulated spectra show an excess over the experimental ones, especially in detectors S1, S2 and S3.

\section{Conclusions}

The necessity of shielding against hadrons and gamma in the ATLAS experiment is crucial. Up to now, there were proposals to use $\mathrm{PE}$ and $\mathrm{Pb}$ or $\mathrm{BPE}$ for shielding. In the present article, the use of a segmented shielding from iron, BPE, iron and LiPE is proposed. The aim of the experimental tests was to show the feasibility and capabilities of such a shielding.

The results obtained from the present experimental tests lead to the following conclusions:

- BPE and LiPE are more effective than PE in decreasing the neutron capture gammaray production ( Table 2, columns 5 and 6 ),

- the use of BPE, compared with LiPE, is slightly favoured from the point of view of 
neutron flux suppression ( Table 2 and Fig. 3 ). However, BPE produces intensive gamma-rays with an energy of $478 \mathrm{keV}$,

- the only advantage of $\mathrm{Pb}$ against Fe is its higher capability to suppress gamma. On the other hand $\mathrm{Pb}$ gives an increased number of neutrons (Table 3 and Fig. 4 ). It can be observed ( Table 3 ) that high suppression of gamma can be already achieved with only $5 \mathrm{~cm}$ of Fe. The use of BPE instead of PE gives the possibility to utilize a layer of $\mathrm{Fe}$ with a thickness comparable to that requested for $\mathrm{Pb}$. This is the consequence of the strong suppression of high energy gamma ( $\mathrm{H}$ and $\mathrm{Fe})$ in $\mathrm{BPE}$ relative to $\mathrm{PE}$ ( Table 2 ). Lower cost and possibly simplified problem of construction are also considerations favouring the choice of Fe shielding.

- the shielding capabilities of BPE and LiPE can be combined. The use of additional LiPE shielding improves the results ( Table 5 ).

Based on the conclusions above, it appears that the best possibility for hadron and gamma shielding for the ATLAS forward region should be a segmented shielding made of outer layers of iron, BPE, iron and, optionally LiPE. The authors think that the use of iron shielding offers the advantages of lower cost and possibly easier construction. The thicknesses of BPE, LiPE and iron layers should be determined from the results of Monte Carlo studies tested against the present measurements and extrapolating the results from CERN-PS to the real ATLAS situation and environment. The thicknesses of the shielding material layers will be also constrained by suppression factor requirements, the mechanical integrity, rigidity and stability of the whole shielding assembly.

The results of our measurements can be also applied to underground experiments such as double beta decay [5]. Although the radiation environment of underground experiments differs from that encountered in ATLAS, the achievement of low backgrounds and in particular the reduction of the background due to neutrons is a necessity for these experiments in order to record useful data.

\section{Acknowledgements}

This work has been conducted as part of the research project "ATLAS experiment at the LHC". This work has been supported by the Ministry of Industry and Trade of the Czech Republic and in part by the Grant Agency of the Czech Republic grant No. 202/98/1216. We would like to thank KOPOS Kolin for production and delivery of lithium doped polyethylene free of charge. One of us (C. L.) thanks NSERC-Canada for their support. We would like also to acknowledge the PS Division Group for their technical support and smooth operation of the beams. The help of F. Lemeilleur, M. Glaser, J. Böhm and E. Ryparová is gratefully acknowledged.

\section{References}

[1] LHC: The Large Hadronic Collider Conceptual design CERN/AC/95-05 (LHC), 20 October 1995.

[2] ATLAS Technical Proposal for a General-Purpose pp Experiment at the Large Hadron Collider at CERN, CERN/LHCC/94-43 (1994).

[3] Y. Fisyak, Study of neutron and $\gamma$ backgrounds in ATLAS, ATLAS Internal Note, CAL-NO-039, CERN (20 April 1994).

[4] J. Kubašta, S. Pospíšil, I. Štekl, V. Vrba, Comparison of efficiencies of neutron shields containing boron and lithium, ATLAS Internal Note, TECH-NO-016, CERN (28 November 1995). 


\begin{tabular}{|c|c|c|}
\hline distance & $\begin{array}{c}\mathrm{Cd}(558 \mathrm{keV}) \\
\text { peak area } \\
{[\text { counts] }}\end{array}$ & $\begin{array}{c}\mathrm{H}(2223 \mathrm{keV}) \\
\text { peak area } \\
{[\text { counts] }}\end{array}$ \\
\hline 10 & $551 \pm 107$ & $1082 \pm 274$ \\
18 & $1351 \pm 143$ & $3030 \pm 282$ \\
25 & $4897 \pm 118$ & $7696 \pm 215$ \\
30 & $5442 \pm 110$ & $10321 \pm 222$ \\
\hline
\end{tabular}

Table 1: Yields of gamma rays relevant to the slow neutron radiative capture for different distances between the proton beam hitting point and the edge of the iron block. Two $\gamma$-rays from neutron capture on $\mathrm{H}$ from $\mathrm{PE}(2223 \mathrm{keV})$ and on $\mathrm{Cd}(558 \mathrm{keV})$ were used to monitor thermal neutrons. A Cd cap was placed on the Ge detector.

\begin{tabular}{|c|c|c|c|c|c|c|}
\hline thickness & $\begin{array}{c}\text { type of } \\
\text { shielding }\end{array}$ & $\begin{array}{c}\mathrm{B} \\
(478 \mathrm{keV})\end{array}$ & $\begin{array}{c}\mathrm{Fe} \\
(847 \mathrm{keV})\end{array}$ & $\begin{array}{c}\mathrm{H} \\
(2223 \mathrm{keV})\end{array}$ & $\begin{array}{c}\text { Fe } \\
(7631 \mathrm{keV}+ \\
+7645 \mathrm{keV}) \\
{[\text { counts] }}\end{array}$ & $\begin{array}{c}\begin{array}{c}\text { number of } \\
\text { slow } \\
\text { neutrons } \\
{[\text { counts] }}\end{array} \\
{[\mathrm{cm}]}\end{array}$ \\
\hline 8 & PE & $5.0 \pm 0.4^{*}$ & $40.4 \pm 2.6$ & $58.7 \pm 1.3$ & $85.6 \pm 4.6$ & $100.0 \pm 1.0$ \\
8 & $\mathrm{LiPE}$ & $4.8 \pm 0.2^{*}$ & $54.9 \pm 1.3$ & $4.5 \pm 0.2$ & $42.0 \pm 1.9$ & $57.1 \pm 0.7$ \\
8 & BPE & $100.0 \pm 0.3$ & $54.6 \pm 1.3$ & $4.0 \pm 0.2$ & $32.0 \pm 1.5$ & $49.2 \pm 0.7$ \\
\hline 16 & PE & $5.9 \pm 0.6^{*}$ & $28.2 \pm 2.0$ & $100.0 \pm 3.0$ & $100.0 \pm 6.1$ & $59.7 \pm 0.7$ \\
16 & LiPE & $4.2 \pm 0.2^{*}$ & $31.6 \pm 0.9$ & $5.1 \pm 0.2$ & $35.7 \pm 1.7$ & $50.5 \pm 0.7$ \\
16 & BPE & $62.9 \pm 0.2$ & $33.6 \pm 0.9$ & $3.5 \pm 0.2$ & $26.0 \pm 1.1$ & $41.2 \pm 0.6$ \\
\hline & $* *$ & $1.7 \pm 0.3$ & $100.0 \pm 2.6$ & $5.5 \pm 0.2$ & $33.4 \pm 2.3$ & $67.6 \pm 0.8$ \\
\hline & $100=$ & 954308 & 127998 & 761447 & 310332 & 12459 \\
\hline
\end{tabular}

Table 2: Relative numbers of photons detected in a photopeak relevant to shield quality assessment (* resulting from boron contained in the surroundings; ** measurement of background only with the iron block without any shielding). The last column contains the number of slow neutrons given by the Si detector with a ${ }^{6} \mathrm{Li}$ converter. The last row contains the numbers of counts corresponding to 100 . The HPGe detector efficiency has been taken into account in columns 3 to 6 .

\begin{tabular}{|c|c|c|c|c|c|}
\hline $\begin{array}{c}\text { type of } \\
\text { gamma-ray } \\
\text { shielding }\end{array}$ & $\begin{array}{c}\mathrm{B} \\
(478 \mathrm{keV})\end{array}$ & $\begin{array}{c}\text { Fe } \\
(847 \mathrm{keV})\end{array}$ & $\begin{array}{c}\mathrm{H} \\
(2223 \mathrm{keV})\end{array}$ & $\begin{array}{c}\text { Fe } \\
(7631 \mathrm{keV}+ \\
+7645 \mathrm{keV}) \\
{[\text { counts] }}\end{array}$ & $\begin{array}{c}\text { number of } \\
\text { slow } \\
\text { neutrons } \\
{[\text { counts] }]}\end{array}$ \\
\hline [counts] $]$
\end{tabular}

Table 3: Comparison of $\mathrm{Fe}$ and $\mathrm{Pb}$ gamma-ray shielding capabilities. The measurements were done with $16 \mathrm{~cm}$ of BPE. The last column contains the number of slow neutrons given by the Si detector with a ${ }^{6} \mathrm{Li}$ converter. 


\begin{tabular}{|c|c|c|c|c|c|}
\hline $\begin{array}{l}\text { thickness } \\
\text { of Fe } \\
\text { shielding } \\
{[\mathrm{cm}]}\end{array}$ & $\begin{array}{c}\mathrm{B} \\
(478 \mathrm{keV}) \\
{[\text { counts] }}\end{array}$ & $\begin{array}{c}\mathrm{Fe} \\
(847 \mathrm{keV}) \\
{[\text { counts] }}\end{array}$ & $\begin{array}{c}\mathrm{H} \\
(2223 \mathrm{keV}) \\
{[\text { counts }]}\end{array}$ & $\begin{array}{c}\mathrm{Fe} \\
(7631 \mathrm{keV}+ \\
+7645 \mathrm{keV}) \\
{[\text { counts }]}\end{array}$ & $\begin{array}{c}\text { number of } \\
\text { slow } \\
\text { neutrons } \\
\text { [counts] }\end{array}$ \\
\hline 0 & $100.0 \pm 0.2$ & $100.0 \pm 1.6$ & $100.0 \pm 4.9$ & $100.0 \pm 4.4$ & $100.0 \pm 1.8$ \\
\hline 2 & $29.3 \pm 0.2$ & $81.0 \pm 1.4$ & $70.4 \pm 3.7$ & $82.4 \pm 3.9$ & $78.1 \pm 1.6$ \\
\hline 5 & $5.2 \pm 0.1$ & $85.6 \pm 1.5$ & $56.1 \pm 3.7$ & $82.9 \pm 4.0$ & $73.3 \pm 1.5$ \\
\hline 8 & $1.1 \pm 0.1$ & $78.0 \pm 1.3$ & $42.7 \pm 3.3$ & $54.9 \pm 3.1$ & $68.3 \pm 1.5$ \\
\hline
\end{tabular}

Table 4: The results of the tests of the shielding capability of iron against gamma (layer $8 \mathrm{~cm}$ thick of BPE, layer of Fe). The last column contains the number of slow neutrons measured with the Si detector with a ${ }^{6} \mathrm{Li}$ converter.

\begin{tabular}{|c|c|c|c|c|c|}
\hline $\begin{array}{l}\text { thickness } \\
\text { of Fe } \\
\text { shielding } \\
\text { [cm] }\end{array}$ & $\begin{array}{c}\mathrm{B} \\
(478 \mathrm{keV}) \\
{[\text { counts }]}\end{array}$ & $\begin{array}{c}\mathrm{Fe} \\
(847 \mathrm{keV}) \\
{[\text { counts] }}\end{array}$ & $\begin{array}{c}\mathrm{H} \\
(2223 \mathrm{keV}) \\
{[\text { counts] }}\end{array}$ & $\begin{array}{c}\mathrm{Fe} \\
(7631 \mathrm{keV}+ \\
+7645 \mathrm{keV}) \\
{[\text { counts }]}\end{array}$ & $\begin{array}{c}\text { number of } \\
\text { slow } \\
\text { neutrons } \\
\text { [counts] }\end{array}$ \\
\hline & - & - & & - & - \\
\hline 2 & $11.6 \pm 0.1$ & $41.7 \pm 1$ & $69.2 \pm 2$ & $82.0 \pm 3.0$ & $74.2 \pm 1.5$ \\
\hline 5 & $2.5 \pm 0.1$ & $39.9 \pm 1.0$ & $49.9 \pm 2.5$ & $70.3 \pm 3.1$ & $88.8 \pm 1.7$ \\
\hline 8 & $1.0 \pm 0.1$ & $39.1 \pm 0.8$ & $44.8 \pm 2.5$ & $57.4 \pm 2.4$ & $66.4 \pm 1.5$ \\
\hline
\end{tabular}

Table 5: The results of the tests of segmented neutron shielding $(8 \mathrm{~cm}$ of BPE, Fe, $8 \mathrm{~cm}$ of LiPE). The data given in this table are normalized to the first row of the previous table in order to show the influence of additional LiPE layer in the neutron shielding. The last column contains the number of slow neutrons measured with the Si detector with a ${ }^{6} \mathrm{Li}$ converter.

\begin{tabular}{|c|c|c|c|c|}
\hline type of neutron & \multicolumn{4}{|c|}{ scintillating detector } \\
\cline { 2 - 5 } shielding & $\mathrm{S} 1$ & $\mathrm{~S} 2$ & $\mathrm{~S} 3$ & $\mathrm{~S} 4$ \\
\hline no shielding & $6.72 \pm 0.11$ & $7.95 \pm 0.12$ & $8.36 \pm 0.13$ & $6.44 \pm 0.11$ \\
PE & $5.40 \pm 0.12$ & $5.79 \pm 0.12$ & $7.08 \pm 0.14$ & $5.29 \pm 0.12$ \\
$\mathrm{BPE}$ & $4.64 \pm 0.14$ & $6.01 \pm 0.16$ & $6.01 \pm 0.16$ & $4.84 \pm 0.14$ \\
$\mathrm{LiPE}$ & $4.53 \pm 0.09$ & $5.93 \pm 0.10$ & $6.31 \pm 0.10$ & $4.53 \pm 0.09$ \\
\hline
\end{tabular}

Table 6: Number of events (per 100 incident protons) with the response of the scintillating detectors S1-S4 above a threshold corresponding to $4 \%$ of mip signal (see text). The results are given for a distance of $15 \mathrm{~cm}$ between the hitting point of the proton beam into the iron block and the edge of the iron block on the side of the scintillating wall. The thickness of the neutron shielding of each type was $8 \mathrm{~cm}$. 


\begin{tabular}{|c|c|c|c|c|}
\hline type of neutron & \multicolumn{4}{|c|}{ scintillating detector } \\
\cline { 2 - 5 } shielding & $\mathrm{S} 1$ & $\mathrm{~S} 2$ & $\mathrm{~S} 3$ & $\mathrm{~S} 4$ \\
\hline no shielding & $2.13 \pm 0.06$ & $2.62 \pm 0.07$ & $2.96 \pm 0.07$ & $2.16 \pm 0.06$ \\
$\mathrm{PE}$ & $2.52 \pm 0.07$ & $2.49 \pm 0.07$ & $2.77 \pm 0.07$ & $2.06 \pm 0.06$ \\
$\mathrm{BPE}$ & $1.75 \pm 0.06$ & $1.77 \pm 0.06$ & $1.89 \pm 0.06$ & $1.52 \pm 0.05$ \\
$\mathrm{LiPE}$ & $1.93 \pm 0.06$ & $2.12 \pm 0.06$ & $2.56 \pm 0.07$ & $1.71 \pm 0.06$ \\
\hline
\end{tabular}

Table 7: Number of events (per 100 incident protons) with the response of the scintillating detectors S1-S4 above a threshold corresponding to $4 \%$ of mip signal (see text). The results are given for a distance of $\sim 25 \mathrm{~cm}$ between the hitting point of the proton beam into the iron block and the edge of the iron block on the side of the scintillating wall. The thickness of the neutron shielding of each type was $8 \mathrm{~cm}$.

[5] Ch. Briancon et al., The high sensitivity double beta decay spectrometer TGV, Nucl. Instr. and Meth. A 372 (1996) 222.

[6] R.L. Branblett, R.I. Ewing and T.W. Bonner, A new type of neutron spectrometer, Nucl. Instr. and Meth. 9 (1960) 1.

[7] M. Kárný, D. Zahálka, J. Heřmanská, Z. Prouza, Bayes-based solution of dosimetric problem and related unfolding of neutron spectra applied to Bonner spectrometer, Radioisotopy 30, 6 (1989) 1.

[8] Basis Aspects of High Energy Particle Interactions and Radiation Dosimetry, ICRU report No. 28, Washington (1978) 48.

[9] S. Pospíšil , B. Sopko, E. Havránková, Z. Janout, J. Koníček, I. Mácha, J. Pavli̊, Si diode as a small detector of slow neutrons, Rad. Prot. Dos. 46, 2 (1993) 115.

[10] M. Lokajíček et al., Scintillator detector "Mu-wall" for measurement of charged particles leakage in the TILECAL prototype, ATLAS Internal Note, ATLAS-TILE-63, CERN (11 August 1995).

[11] C.W. Fabjan, Calorimetry in High-Energy Physics, Experimental Techniques in HighEnergy Nuclear and Particle Physics, Ed. T. Ferbel, World Scientific (Singapore) $1991,257$.

[12] GEANT, Detector Description and Simulation Tool, CERN Program Library, long writeup W5013, 1994. 


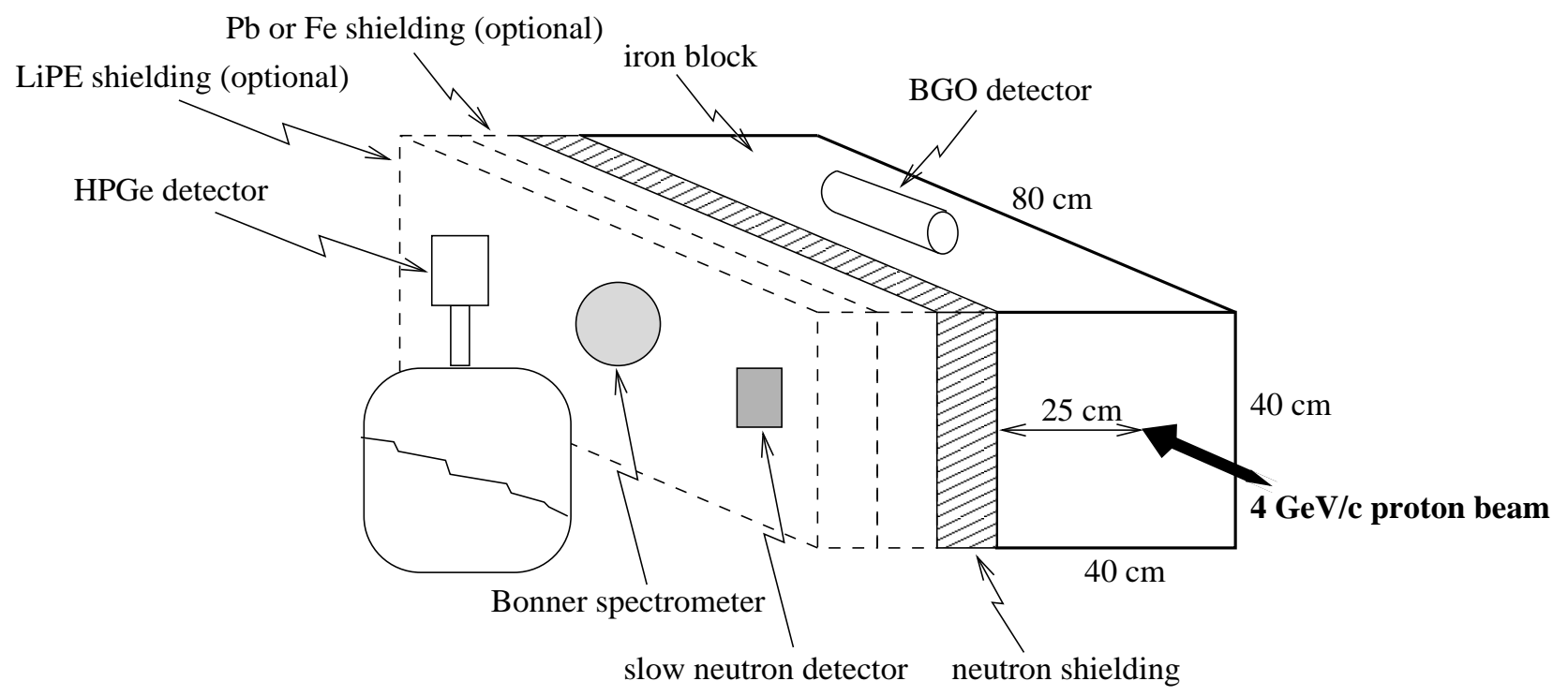

Figure 1: Experimental set-up. 


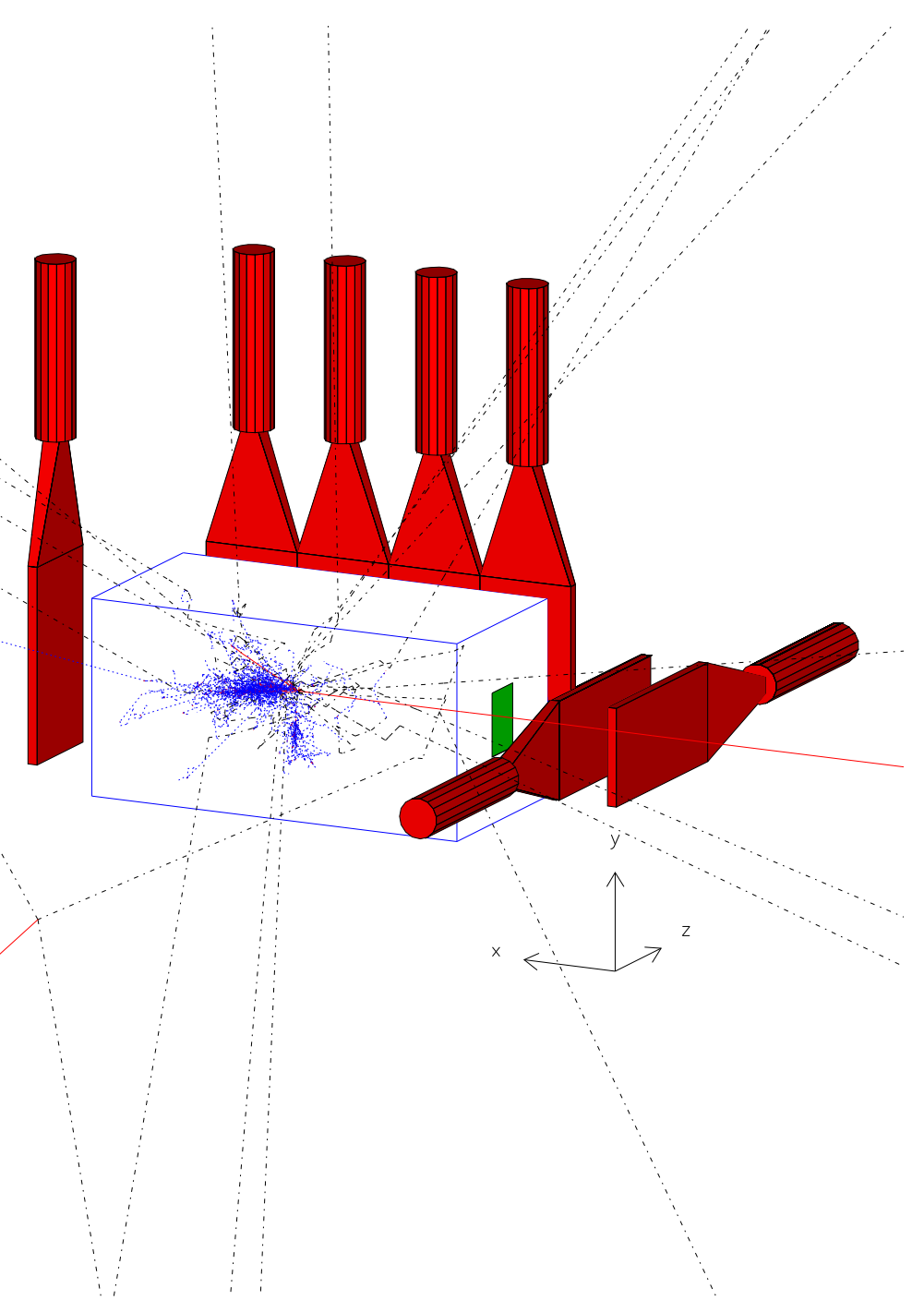

Figure 2: A simulation of a secondary particle cascade produced by $4 \mathrm{GeV} / \mathrm{c}$ incoming proton in the $40 \mathrm{~cm} \times 40 \mathrm{~cm} \times 80 \mathrm{~cm}$ iron block with the arrangment of the scintillator detectors. 


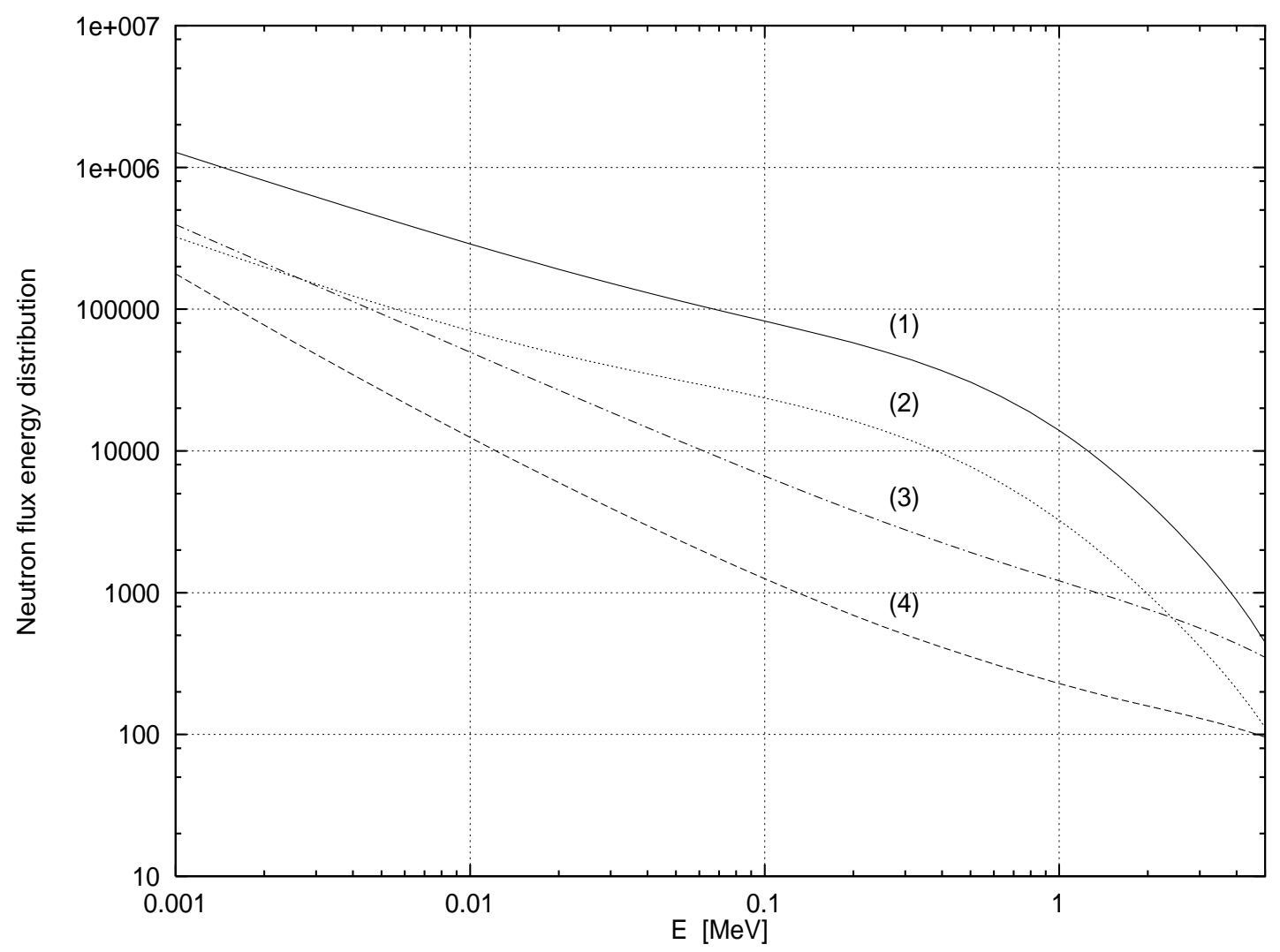

Figure 3: Comparison of the neutron flux energy distribution for neutron shielding materials ( PE, BPE, LiPE) with non-shielded iron (1). The thickness of the neutron shielding materials was $16 \mathrm{~cm}$ in all cases: LiPE (2), BPE (3), PE (4). 


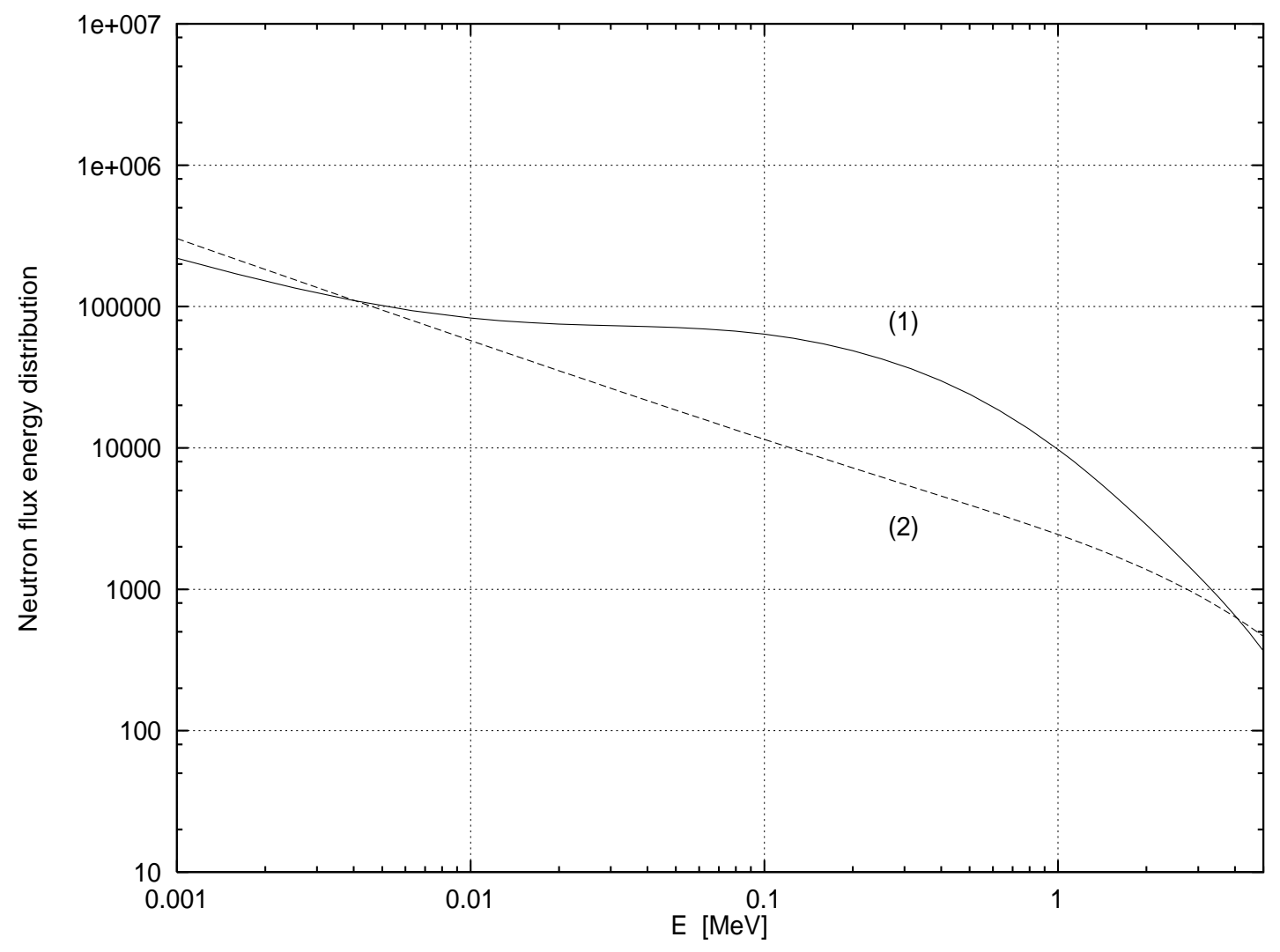

Figure 4: Comparison between neutron shielding based on $\mathrm{PE}$ and $5 \mathrm{~cm}$ of $\mathrm{Pb}(1)$ and shielding based on $\mathrm{BPE}$ and $5 \mathrm{~cm}$ of $\mathrm{Fe}(2)$. The shielding based on $\mathrm{PE}$ and $5 \mathrm{~cm}$ of $\mathrm{Pb}$ was originally proposed in [2]. 


\section{Comparison of simulations and exper. data}
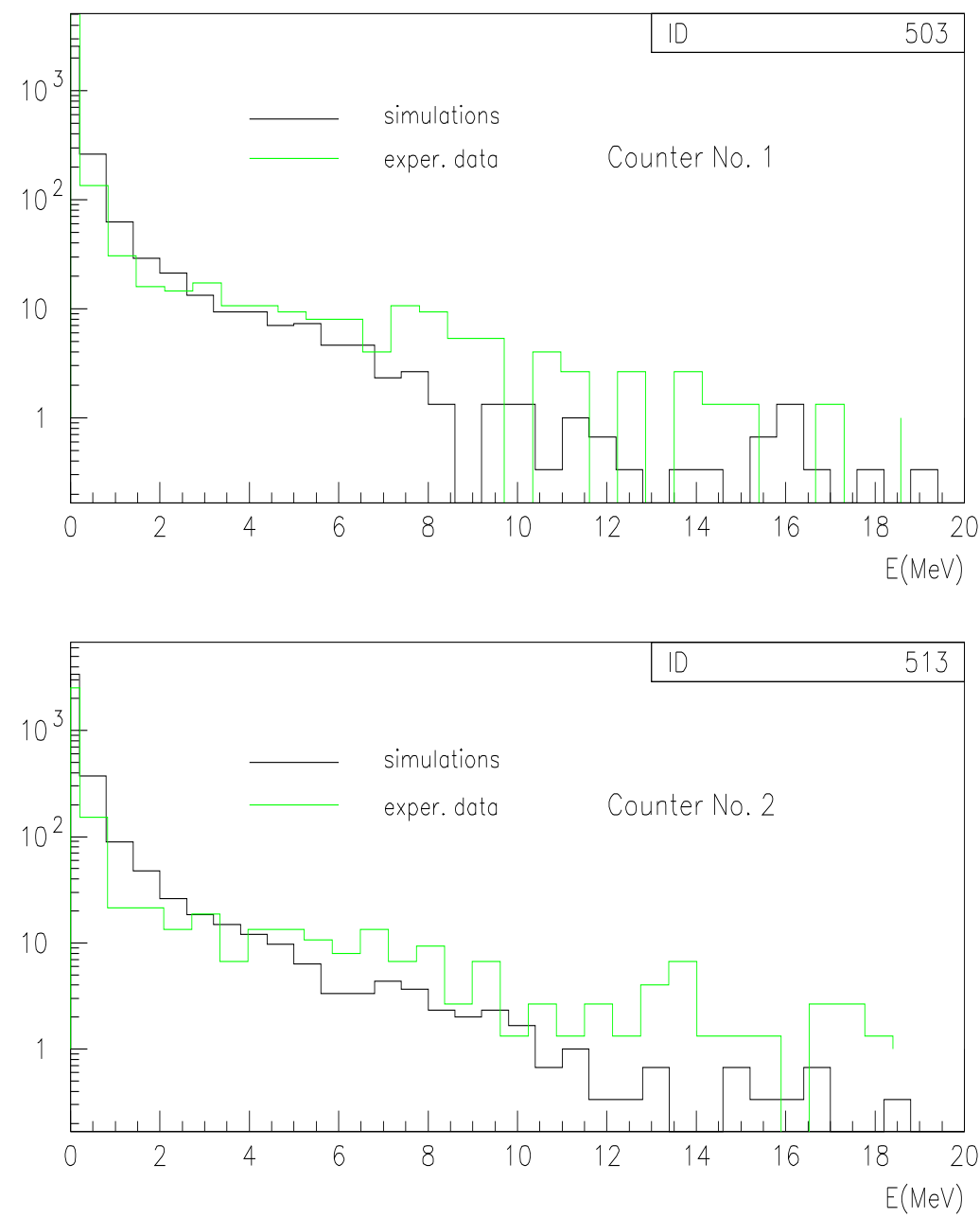

Figure 5: Comparison of the experimental responses in the scintillator detectors S1 and S2 with a GEANT simulation, for a $5 \mathrm{GeV} / \mathrm{c}$ proton beam and an iron block thickness of $20 \mathrm{~cm}$. 


\section{Comparison of simulations and exper. data}
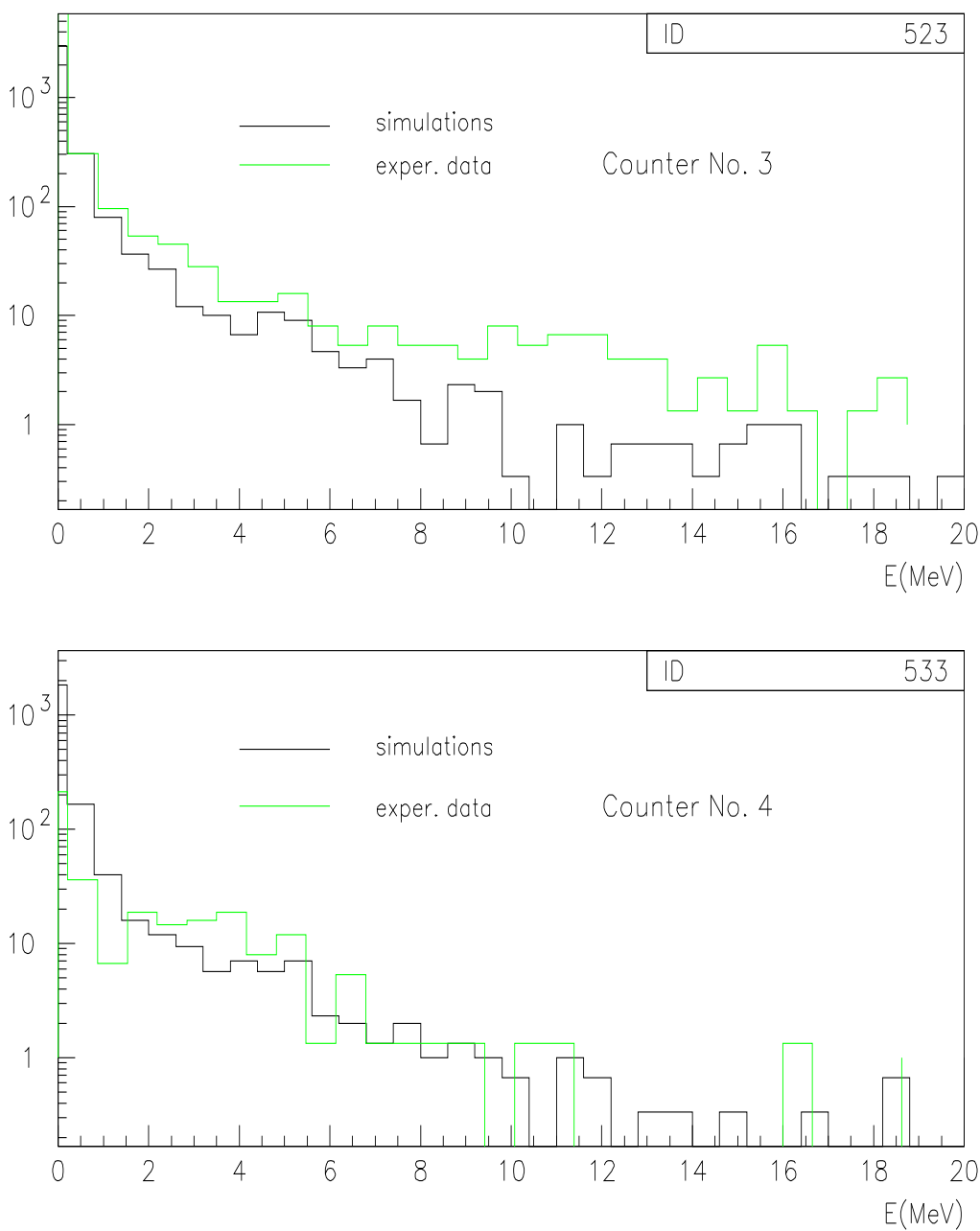

Figure 6: Comparison of the experimental responses in the scintillator detectors $\mathrm{S} 3$ and $\mathrm{S} 4$ with a GEANT simulation, for a $5 \mathrm{GeV} / \mathrm{c}$ proton beam and an iron block thickness of $20 \mathrm{~cm}$. 